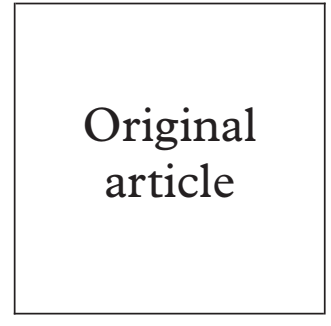

\title{
Do the factors associated with successful contact tracing of patients with gonorrhoea and chlamydia differ?
}

\author{
J D C Ross, A Sukthankar, K W Radcliffe, J Andre
}

\begin{abstract}
Objective: To assess and compare factors which may be associated with successful contact tracing in patients with gonorrhoea and chlamydia.

Study design: Prospective observational study of patients attending a genitourinary medicine clinic with a diagnosis of gonorrhoea or chlamydia. Multivariate analysis model including demographic, socioeconomic, and behavioural variables.

Results: The attendance of at least one sexual contact was associated with naming more contacts for patients with gonorrhoea (OR 1.44, 95\% CI 1.04-2.01). A history of gonorrhoea was associated with successful contact tracing for patients with chlamydia (OR 1.46, 95\% CI 1.12-1.9). Successful contact tracing, as defined by at least one confirmed contact attendance after the index case, was not associated with age, sex, sexual orientation, history of chlamydia, use of condoms, marital status, ethnicity, or socioeconomic status for either gonorrhoea or chlamydia.

Conclusions: Differences in the composition of the core groups infected with gonorrhoea and chlamydia are not explained by differences in contact tracing success. In the clinic setting studied, the outcome of contact tracing was not associated with a variety of demographic, socioeconomic, and behaviour factors.

(Sex Transm Inf 1999;75:112-115)
\end{abstract}

Keywords: gonorrhoea; chlamydia; contact tracing; epidemiology; core groups

\section{Introduction}

Gonorrhoea and chlamydia continue to be responsible for considerable morbidity despite available, effective, and relatively cheap curative therapy. In the United Kingdom the network of genitourinary medicine clinics provides free diagnosis and treatment to those infected with sexually transmitted diseases and attempts to trace the sexual contacts of those found to be infected. Despite this integrated and accessible approach the incidence of gonorrhoea and chlamydia is increasing. ${ }^{1}$

Although both gonorrhoea and chlamydia are acquired through sexual contact they differ in a number of respects. Chlamydia is three times as common as gonorrhoea, ${ }^{1}$ has a longer period of infectivity, and is less infectious than gonorrhoea. ${ }^{2}$ Data from the United States suggest that those who acquire gonorrhoea tend to be older, live within a limited number of geographical areas, and belong to "black" ethnic groups when compared with patients with chlamydia. ${ }^{3}$ Given these differences should the approach to contact tracing be different for the two conditions?

This study compares sociodemographic variables in patients with gonorrhoea and chlamydia, and attempts to identify factors which may influence the success of contact tracing.

J D C Ross

A Sukthankar

K W Radcliffe

$\mathrm{J}$ Andre

Correspondence to: Dr J D C Ross, Whittall

Street Clinic, Whittall Street, Birmingham B4 6DH.

Accepted for publication 9 December 1998
All patients attending the Whittall Street Clinic, Birmingham between 1 January 1997 and 31 October 1997 with a diagnosis of gonorrhoea or genital chlamydial infection were evaluated prospectively. The Whittall Street
Clinic is a large urban genitourinary medicine clinic with 27000 attendances per year. Patients attending the clinic were screened for Neisseria gonorrhoeae by taking a urethral swab in men and endocervical and urethral swabs in women. Samples were taken from the pharynx and rectum as indicated by the clinical history and the diagnosis of gonorrhoea was made on the basis of a positive culture from the swab on modified Thayer-Martin medium. The clinic procedure for chlamydia testing changed during the study period-the Dako enzyme linked immunoassay (EIA) being used until 28 May 1997 and the Abbott LCX ligase chain reaction (LCR) assay thereafter. Men were offered screening by providing a first pass urine sample, or a urethral swab was obtained. The samples were then tested by either EIA or LCR. An endocervical swab was used to screen women for chlamydia using either EIA or LCR.

Information on age, sex, ethnic origin (as allocated by the patient) and postcode of the patient was collected during clinic registration. Information on sexual behaviour was obtained by medical staff during the clinical interview comprising patient history of previous gonorrhoea or chlamydia; number of sexual contacts; sexual orientation based on the most recent sexual contact; use of condoms. The time period for inclusion of sexual contacts was varied according to the diagnosis and the sex of patient to reflect differences in the incubation period between gonorrhoea and chlamydia, and likely periods of infectiousness. For gonorrhoea sexual contacts within 3 months for women and 1 month for men were included, 
Table 1 Univariate analysis of demographic and behavioural factors in relation to successful contact tracing, gonorrhoea

\begin{tabular}{|c|c|c|c|}
\hline & $\begin{array}{l}\text { Contact tracing } \\
\text { unsuccessful }\end{array}$ & $\begin{array}{l}\text { Contact tracing } \\
\text { successful }\end{array}$ & $O R(95 \% C I)$ \\
\hline Age under 25 & 63 & 25 & $1.3(0.7-2.4)$ \\
\hline Age 25 or over & 71 & 37 & \\
\hline Black Caribbean & 80 & 37 & \\
\hline White & 46 & 17 & $0.8(0.4-1.6)$ \\
\hline Jarman score above median (43) & 56 & 31 & \\
\hline Jarman score below/equal to median & 61 & 24 & $0.7(0.4-1.4)$ \\
\hline $\begin{array}{l}\text { Married/co-habiting/ } \\
\text { divorced/separated }\end{array}$ & 12 & 11 & \\
\hline Single & 122 & 51 & $0.5(0.2-1.1)$ \\
\hline Male & 94 & 50 & \\
\hline Female & 40 & 12 & $0.6(0.3-1.2)$ \\
\hline Condoms used mostly or always & 39 & 20 & \\
\hline Condoms used never or occasionally & 95 & 42 & $0.9(0.4-1.7)$ \\
\hline More than one contact named & 39 & 30 & \\
\hline One contact named & 95 & 32 & $0.4(0.2-0.8)$ \\
\hline No history of gonorrhoea & 88 & 43 & \\
\hline History of gonorrhoea & 46 & 19 & $0.8(0.4-1.6)$ \\
\hline No history of chlamydia & 103 & 43 & \\
\hline History of chlamydia & 31 & 19 & $1.5(0.7-2.9)$ \\
\hline No additional STD diagnosed & 116 & 55 & \\
\hline Additional STD diagnosed & 18 & 7 & $0.8(0.3-2.1)$ \\
\hline Heterosexual & 115 & 55 & \\
\hline Homosexual & 19 & 7 & $0.8(0.3-1.9)$ \\
\hline
\end{tabular}

Data missing for ethnic group $(n=1)$ and Jarman scores $(n=24)$. Odds ratio calculated for ethnic groups white and black Caribbean only.

Table 2 Univariate analysis of demographic and behavioural factors in relation to successful contact tracing, chlamydia

\begin{tabular}{|c|c|c|c|}
\hline & $\begin{array}{l}\text { Contact tracing } \\
\text { unsuccessful }\end{array}$ & $\begin{array}{l}\text { Contact tracing } \\
\text { successful }\end{array}$ & OR $(95 \% C I)$ \\
\hline Age under 25 & 146 & 62 & \\
\hline Age 25 or over & 132 & 77 & $1.4(0.9-2.1)$ \\
\hline Black Caribbean & 95 & 60 & \\
\hline White & 150 & 69 & $0.7(0.5-1.2)$ \\
\hline Jarman score above median (43) & 87 & 51 & \\
\hline Jarman score below/equal to median & 155 & 75 & $0.8(0.5-1.3)$ \\
\hline $\begin{array}{l}\text { Married/co-habiting/ } \\
\text { divorced/separated }\end{array}$ & 29 & 20 & \\
\hline Single & 249 & 119 & $0.7(0.4-1.3)$ \\
\hline Male & 131 & 63 & \\
\hline Female & 147 & 76 & $1.1(0.7-1.6)$ \\
\hline Condoms used mostly or always & 69 & 23 & \\
\hline Condoms used never or occasionally & 209 & 116 & $1.7(1-2.8)$ \\
\hline More than one contact named & 124 & 48 & \\
\hline One contact named & 154 & 91 & $1.5(1-2.3)$ \\
\hline No history of gonorrhoea & 235 & 102 & \\
\hline History of gonorrhoea & 43 & 37 & $2(1.2-3.3)$ \\
\hline No history of chlamydia & 206 & 94 & \\
\hline History of chlamydia & 72 & 45 & $1.4(0.9-2.1)$ \\
\hline No additional STD diagnosed & 233 & 111 & \\
\hline Additional STD diagnosed & 45 & 28 & $1.3(0.8-2.2)$ \\
\hline Heterosexual & 275 & 139 & \\
\hline Homosexual & 3 & 0 & $\mathrm{n} / \mathrm{a}$ \\
\hline
\end{tabular}

Data missing for Jarman scores $(n=49)$. Odds ratio calculated for ethnic groups white and black Caribbean only.

while for chlamydia the time periods were 6 months for women and 3 months for men. The presence of any additional sexually transmitted infections, defined for the purposes of the study as comprising syphilis, genital herpes, genital warts, or Trichomonas vaginalis was noted. The Jarman 8 score, as a marker of socioeconomic status, was taken as that allocated to each patient's postcode at the 1991 population census. The calculation of the Jarman index is shown in the appendix.

Tracing of sexual contacts was undertaken by the clinic health advisers who were aware that the study was taking place. The patient was either given a contact slip to pass on to his/her partner(s) requesting them to make an appointment at the clinic, or else direct communication was made with the named partner by the clinic. The method of contact tracing did not differ between those diagnosed with gonorrhoea or chlamydia. Successful contact tracing for each disease episode was defined as the confirmed attendance of a sexual contact occurring at least 24 hours after the index case. Where the contact did not attend the Whittall Street Clinic confirmation of attendance elsewhere was obtained verbally by phone or by letter. Those attending as a result of contact tracing who were found to be infected were counted as new index cases if they named additional contacts.

A power calculation suggested that a sample size of 348 would detect a difference in the contact tracing success rate of $5 \%$ (33\% to $38 \%$, RR 1.15) with $80 \%$ power.

Data were entered into the ACCESs database package (Microsoft) and subsequent analysis performed in the sPSS statistical package (SPSS Inc). Univariate comparisons were made by calculating odds ratios with $95 \%$ confidence intervals. The multivariate comparison was made using a forward conditional logistic regression model with entry into the model when $\mathrm{p}<0.05$ and removal when $\mathrm{p}>0.1$.

\section{Results}

A total of 826 patient episodes of gonorrhoea or chlamydia were diagnosed during the study period. Three denied any contacts within the protocol period, 107 had a dual infection with both gonorrhoea and chlamydia, and $103 \mathrm{had}$ only one named contact who had attended before the "index case". Excluding these individuals, 196 episodes of gonorrhoea were diagnosed in 193 patients and 417 episodes of chlamydia in 405 patients. The patients with chlamydia who were screened using EIA did not differ from those screened using LCR with respect to number of contacts, age, Jarman index, ethnicity, sexual orientation, or condom use.

Overall contact tracing was successful, as defined by the study protocol, in 32\% (62/196) of patient episodes of gonorrhoea and 33\% (139/417) of chlamydial episodes. On univariate analysis of patients with gonorrhoea, only the naming of more than one sexual contact was associated with at least one contact attending (table 1). For those with chlamydia a history of gonorrhoea was associated with successful contact tracing (table 2). On multivariate analysis these associations were maintained: naming of more than one sexual contact in those with gonorrhoea OR 1.44 (95\% CI 1.04-2.01) and history of gonorrhoea in those with chlamydia OR 1.46 (95\% CI 1.12-1.9).

\section{Discussion}

The core group theory ${ }^{4}$ for transmission of gonorrhoea proposes that a small number of individuals maintain infection within a community by repeatedly becoming infected and having numerous partners both within and outside the core group. The characteristics of the core group may include black racial origin and a low level of education. ${ }^{5}$ If individuals in the core group could be identified and treated the incidence of infection should fall and, in theory, the disease will eventually be eradicated. Thus contact tracing to identify, screen, and treat this group is of particular importance. 
There is evidence however that the demographics of the core groups differ for gonorrhoea and chlamydia and, in addition, individuals infected with gonorrhoea may live within a smaller number of geographically defined areas compared with a more diffuse residence pattern for those with chlamydia, suggesting different sexual mixing patterns. ${ }^{36}$ Thus the core group membership and their sexual interactions may vary for gonorrhoea and chlamydia.

The tracing of sexual contacts has been undertaken in many countries in an attempt to prevent reinfection of the index patient, treat infected partners, and reduce the burden of infection in the community. ${ }^{7}$ Although intrinsically appealing, it has proved difficult to prove objectively that contact tracing achieves its aims at a population level. The identification of factors influencing the success of contact tracing may help target available resources more effectively.

Our results suggest that successful contact tracing for patients with gonorrhoea or chlamydia was not associated with age, sex, sexual orientation, history of chlamydia, use of condoms, marital status, ethnicity, or socioeconomic status. Despite some of these variables being markers for core group membership our results suggest that differences in the success of contact tracing do not contribute to the establishment or maintenance of core groups. Successful contact tracing was more likely for patients with gonorrhoea who named more sexual contacts but, since the protocol definition of success was the confirmed attendance of at least one partner, this is not unexpected. For patients with chlamydia a history of gonorrhoea was associated with an improved contact tracing success which may reflect prior experience of sexually transmitted infections resulting in a change in behaviour with a greater effort to encourage contacts to attend.

We chose to trace sexual contacts of patients over varying time periods dependent on the diagnosis and sex of the index case to reflect the differences in incubation periods for the two infections and the greater likelihood of prolonged asymptomatic infection in women. The resulting longer contact tracing period for chlamydia infections may explain why similar numbers of contacts were named by patients with gonorrhoea and chlamydia, despite previous studies suggesting that those infected with gonorrhoea have more partners. ${ }^{6}$ It has also been suggested that men from a black ethnic background identify more partners than black women. ${ }^{6}$ When sex, ethnic origin, and number of partners named were included in our multivariate analysis model none was found to be independently associated with the successful tracing of sexual contacts.

The contact tracing success rate in this study, $32 \%$ of gonorrhoea patient contacts and $33 \%$ of chlamydia patient contacts, is lower than some other published figures. ${ }^{69}$ This may reflect our stringent definition of successful contact tracing which ensured that only those contacts who could reasonably attended as a result of the contact tracing process were included. Contacts who attended before the index case were excluded, as were attendances at a genitourinary medicine clinic which could be not be verified. Future studies of contact tracing could benefit from a standardised definition of success.

Jarman 8 scores were used as an estimate of socioeconomic status based on the score assigned to the patient's post code in the 1991 census. Since the Jarman score includes non-white ethnic group as one of its variables the potential arises for confounding with the patient's self assigned ethnic origin. Although possible this is unlikely because ethnic group is only one of the eight variables used to calculate the Jarman score, and is weighted lower than the other seven variables (see appendix).

Where successful contact tracing is defined as the attendance of at least one identified sexual contact only the total number of sexual contacts increased the success rate for patients with gonorrhoea, and a history of gonorrhoea was associated with improved success for those with chlamydia. Contact tracing success was not associated with other demographic, socioeconomic and behaviour factors for patients with either infection. Variations in contact tracing do not appear to explain the differences in the core groups for gonorrhoea and chlamydia, nor their differing incidences. It would therefore seem inappropriate to target particular patient groups for more "intensive" contact tracing. There is a need for a standardised definition of successful contact tracing to aid future research and audit.

Individual contributions from authors not available.

\section{Appendix}

JARMAN 8 VARIABLES FROM 1991 CENSUS

The following variables are extracted from the 1991 census:

$\mathrm{V} 1=$ lone pensioners as percentage of all residents in households

$\mathrm{V} 2=$ under 55 as percentage of all residents in households

V3 = persons in single parent households as percentage of all residents in households

$\mathrm{V} 4=$ persons in households headed by a person in socioeconomic group 11 (unskilled) as percentage of all residents in households

V5 = unemployed as percentage of economically active residents, aged 16 and over

V6 $=$ persons in households with more than one person per room as percentage of all residents in households

$\mathrm{V} 7=$ persons changed address in last year as of all residents

V8 $=$ persons in non-white ethnic groups as percentage of all residents

\section{CALCULATION OF JARMAN 8 SCORES}

Normalise variables by transforming variables.

All variables transformed using $\mathrm{LN}(\mathrm{V}+\mathrm{L})$.

NB: It should be noted that although the above transformations improved the normality of the distributions of the census variables, the distributions still remained significantly nonnormal in some cases. 
Standardise transformed variables by subtracting the mean and dividing by the standard deviation.

$$
\text { eg, } \mathrm{S} 1=(\mathrm{N} 1-\text { mean of } \mathrm{N} 1) / \mathrm{SD} \text { of } \mathrm{N} 1
$$

Calculate Jarman scores by calculating a weighted sum of the standardised variables

ie, Jarman $8=(6.62 \times \mathrm{s} 1)+4.64 \times \mathrm{s} 2)$ $(3.01 \times \mathrm{s} 3)+(3.74 \times \mathrm{s} 4)+(3.34 \times \mathrm{s} 5)+(2.88 \times \mathrm{s} 6)$ $+(2.68 \times \mathrm{s} 7)+(2.50 \times \mathrm{s} 8)$

Note: Jarman scores for wards are standardised by regional figures, hence the scores show deprivation relative to all the other wards in the region.

1 Simms I, Hughes G, Swan AV, et al. New cases seen at genitourinary medicine clinics: England 1996. Commun Dis Rep Suppl 1998;1:S1-S11.

2 Lycke E, Lowhagen GB, Hallhagen G, et al. The risk of transmission of genital Chlamydia trachomatis infection is less than that of genital Neisseria gonorrhoeae infection. Sex Transm Dis 1980;7:6-10

3 Zimmerman HL, Potterat JJ, Dukes RL, et al. Epidemiologic differences between chlamydia and gonorrhoea. Am $\mathcal{F}$ Public Health 1990;80:1338-42.

4 Yorke JA, Hethcote HW, Nold A. Dynamics and control of the transmission of gonorrhoea. F Am Vener Dis Assoc 1978; 5:51-6.

5 Brooks GF, Darrow WW, Day JA. Repeated gonorrhea: an analysis of importance and risk factors. If Infect Dis 1978;137:161-9.

6 Katz BP, Caine VA, Jones RB. Evaluation of field follow-up in a sexually transmitted disease clinic for patients at risk for infection with Neisseria gonorrhoeae and Chlamydia trachomatis. Sex Transm Dis 1992;19:99-103.

7 Cowan FM, French R, Johnson AM. The role and effectiveness of partner notification in STD control: a review. Geniness of partner notification in
tourin Med 1996;72:247-52.

8 David LM, Wade AAH, Natin D, et al. Gonorrhoea in Coventry 1991-1994: epidemiology, coinfection and evaluation of partner notification in the STD clinic. Int $\mathcal{F} S T D$ AIDS 1997;8:311-16.

9 McLean K. Audit of contact tracing for gonococcal and chlamydial infection. Int $f$ STD AIDS 1993;4: 181. 